\title{
Preclinical Study on the Ameliorating Effect of L- Ascorbic Acid for the Oxidative Stress of Caused by Chronic Administration of Organic Nitrates in Cardiovascular Diseases
}

\author{
Ahmed M Hamdan ( $\nabla$ dr_ahmed_m_hamdan@yahoo.com ) \\ university of tabuk https://orcid.org/0000-0003-4930-792X \\ Zuhair M. Mohammedsaleh \\ University of Tabuk \\ Aalaa Aboelnour \\ University of Mansoura \\ Sherif M.H. Elkhannishi \\ University of Mansoura
}

\section{Research Article}

Keywords: Methemoglobin, Organic nitrates, Oxidative stress, Vitamin C

Posted Date: October 19th, 2021

DOl: https://doi.org/10.21203/rs.3.rs-968191/v1

License: (c) (1) This work is licensed under a Creative Commons Attribution 4.0 International License.

Read Full License

Version of Record: A version of this preprint was published at Saudi Pharmaceutical Journal on October 1st, 2022. See the published version at https://doi.org/10.1016/j.jsps.2022.07.001. 


\section{Abstract}

\section{Purpose}

The therapeutic activity of Glyceryl trinitrate (GTN) is mainly regulated by liberating nitric oxide (NO) and reactive nitrogen species (RNS). During this biotransformation, oxidative stress and lipid peroxidation inside the red blood cells (RBCs) occur. The principal objective of our research is to explain the ameliorating effect of L-ascorbic acid for the deleterious effects of chronic administration of nitrovasodilator drugs.

\section{Methods}

We studied some biochemical parameters for the oxidative stress using groups of high sucrose/fat (HSF) diet Wistar male rats chronically orally administered ISMN. Afterwards, we evaluated the role of Lascorbic acid against these biochemical changes.

\section{Results}

Chronic treatment with organic nitrates caused elevated serum levels of lipid peroxidation, hemoglobin derivatives as methemoglobin and carboxyhemoglobin, rate of hemoglobin autoxidation, the cellular levels of pro-inflammatory cytokines marker (NF-kB) and apoptosis markers (caspase-3) in myocardium muscles in a dose dependent manner. Meanwhile, such exposure caused decline in the enzymatic effect of superoxide dismutase (SOD), glutathione (GSH) and catalase activity (CAT) accompanied with a decrease of in the level of mitochondrial oxidative stress marker (nrf2) in myocardium muscles and decrease in the serum iron and total iron binding capacity (TIBC) in a dose dependent manner. Concomitant treatment with L-ascorbic acid significantly diminished these changes for all examined parameters.

\section{Conclusion}

Chronic administration of organic nitrates leads to the alteration of the level of oxidative stress factors in the myocardium tissue due to generation of reactive oxygen species. Using vitamin $\mathrm{C}$ can effectively ameliorate such intoxication to overcome the nitrate tolerance.

\section{Introduction}

Hypertension, congestive heart failure, angina pectoris, ischemic heart disease and cerebrovascular disease are the worldwide main reason of deaths and aliment adjusted life years [1, 2]. Isosorbide-5mononitrate (ISMN) is an organic nitrate vasodilator which represents a promising therapeutic option for both treating and preventing such cardiovascular diseases. On the molecular level, Glyceryl trinitrates (GTNs), including the organic nitroglycerins, act as pro-drugs for nitric oxide which is a highly effective vasodilator drug. Bioactivation of nitrate occurs when inert nitrite converted to nitric oxide (NO) by mitochondrial aldehyde dehydrogenase-2 (ALDH-2) [3, 4]. Nitric oxide (NO) plays an important rule for 
relaxing the vascular smooth muscle and increases blood flow to the myocardium [5]. GTNs are recommended for treatment of stable coronary artery disease that is still symptomatic despite treatment with aspirin, beta-adrenergic receptor blockers or ACE-inhibitors [6].

The use of GTN is widely limited by its chronic administration procedure due to the rapid emergence of tolerance and/or endothelial dysfunction. In most cases, tolerance is explained by the increased intracellular oxidative stress. The anti-ischemic effect of GTNs is rapidly lost with chronic administration of low-doses. This phenomena is explained by the reactions of reactive RNS; NO, peroxynitrite and $\mathrm{NO}_{2}$, with lipids and other biomolecules under increased intracellular oxidative stress $[7,8]$. The undesirable and poorly tolerated hemodynamic side-effects of GTNs such as headache and orthostatic hypotension can often occur owing to the systemic vasodilatation [9].

Treatment with GTNs raises the cellular level of generated reactive oxygen species (ROS) within the vascular tissue. Superoxide dismutase (SOD), catalase and glutathione (GSH) are cellular defense mechanisms providing protection against the damage effect of free radicals and reactive oxygen species (ROS) [10]. GTNs chronic administration leads to biotransformation into NO in the reticulocytes of the vascular tissues which in turn causes oxidative stress, SOD inhibition, depletion of GSH pool and decreased bioavailability of NO [11].

Tolerance due to chronic administration of GTNs, reduction of its clinical response, leads to the urgent need of increasing the GTNs dosages in order to maintain the vasodilating effect [12]. The molecular mechanism of this tolerance includes that GTNs interferes the purine metabolism and drains intracellular ATP and GTP. GTNs blocks xanthine oxidase (XO), a rate limiting enzyme for the metabolic bioactivation of GTNs into NO. L-ascorbic acid prevented of XO blockage which results in an increased NO production from GTN $[13,14]$. Nitrate tolerance is handled by generating a 12-h nitrate-free interval. Although this strategy can manage nitrate tolerance, it resulted in increased risk of cardiovascular problems during these free periods, especially in the early light phase [14].

NO metabolism mainly occurs in the red blood cells (RBCs) $[15,16]$. Besides, NO bioactivity is preserved by RBCs. Metabolism of GTNs leads to the formation of inorganic nitrates that can oxidize hemoglobin into methemoglobin [11].

Organic nitrates cause oxidation of hemoglobin $(\mathrm{Hb})$ resulted in methemoglobin (MetHb) formation and $\mathrm{O}_{2}$ liberation. This autoxidation reaction of oxyhemoglobin $(\mathrm{oxyHb})$ is super rapid [17]. Such elevated methemoglobin (metHb) content is a dose-dependent of the organic nitrates concentration [18]. MetHb production catalysis binding of oxygen to normal hemoglobin, so less oxygen will be available at the tissue level [19].

Iron is present in the ferric state $\left(\mathrm{Fe}^{3+}\right)$ in methemoglobin, so it cannot bind to oxygen. This procedure is irreversible resulting in NO oxidation to nitrate and NO resistance. Subsequently, serious hypertension, lipid peroxidation, and vascular injury are followed. Both cellular oxidative stress and inflammatory tissue injury can be occurred due to the released heme and globin as a result of the catabolism of NO and/or 
oxyhemoglobin and methemoglobin [20,22]. GTN tolerance is accompanied by raising the cellular level of free radical-induced lipid peroxidation [23]. Nitrate tolerance has been considered to be a limitation of nitrate therapy. The effectiveness and benignity of nitrate therapy could be dramatically improved (two to three times) upon concomitant administration of antioxidants which prevents the need for nitrate-free intervals $[7,13]$.

Ascorbic acid is a potent electron donor water-soluble antioxidant. It efficiently preserves the body functions against oxidative stress and is a cofactor in numerous essential enzymatic reactions [24]. Ascorbic acid enhances endothelial vasodilation and prevents endothelial dysfunction $[25,26]$. Antioxidant agents are responsible to convert methemoglobin back to hemoglobin [26].

\section{Material And Methods}

\section{Animal treatment}

As previously described in [27], Forty-two 14-week-old male Wistar rats High Sucrose/Fat (HSF) fed (body weight, $403.19 \pm 18.12 \mathrm{~g}$ ) obtained from the Animal House facilities, University of Tabuk, Saudi Arabia were divided into seven groups in a random manner. Rats were placed in conventional cages (3 rats/cage). The High Ssucrose/Fat diet consisted of $79 \%$ normal diet, $10 \%$ sucrose, $5 \%$ lard, $5 \%$ cholesterol, and $1 \%$ lithocholic acid. They were kept for two weeks before the experiments started in rooms with controlled temperature $\left(23 \pm 2^{\circ} \mathrm{C}\right)$ and humidity $(50 \pm 5 \%)$ and $12 / 12 \mathrm{~h}$ light/dark cycle starting at 6:00 am. Both their weight and their caloric intake were recorded weekly. The methods got the approval by the ethical research project committee in University of Tabuk (UT-46-10-2018) and all steps were performed in accordance with its relevant guidelines and regulations. All animal fed with a high sucrose/fat (HSF) diet for twelve weeks. Isosorbide-5-mononitrate (ISMN) was dissolved in water and given orally at three different doses $(0.3 \mathrm{mg} / \mathrm{kg}, 0.6 \mathrm{mg} / \mathrm{kg}$, and $1.2 \mathrm{mg} / \mathrm{kg}$ body weight). Oral L-ascorbic acid was dissolved in water and given orally $(20 \mathrm{mg} / \mathrm{kg}$ body weight).

\section{Animals were divided into seven main groups as follow:}

Group (1): Control group which fed with HSF only.

Group (2) was given ISMN $0.3 \mathrm{mg} / \mathrm{kg}$ orally.

Group (3) was given ISMN $0.3 \mathrm{mg} / \mathrm{kg}$ followed by oral dose of L-ascorbic acid.

Group (4) was given ISMN 0.6 mg/ kg orally.

Group (5) was given ISMN $0.6 \mathrm{mg} / \mathrm{kg}$ followed by oral dose of L-ascorbic acid.

Group (6) was given ISMN $1.2 \mathrm{mg} / \mathrm{kg}$ orally.

Group (7) was given ISMN $6.8 \mathrm{mg} / \mathrm{kg}$ followed by oral dose of L-ascorbic acid. 


\section{Blood Sample collection}

Blood samples were drained from each rat in each group and centrifuged for isolation of the serum. Samples were stored at $-70 \mathrm{C}$.

The following investigations were done:

\section{Hematological parameters:}

Methemoglobin, Carboxyhemoglobin, oxygen saturation, and oxygen content were measure using Arnaud et al and Boumba et al $[28,29]$.

Briefly, Methemoglobin is measured spectrophotometrically at $540 \mathrm{~nm}$ after complete transformation into Cyanmethemoglobin ( $\mathrm{CnMetHb})$ using potassium cyanide [28]. Carboxyhemoglobin $(\mathrm{COHb})$ was assayed spectrophotometrically with automated multi-wavelength method [29]. A double-beam Shimadzu model UV-1900i UV-Vis Spectrophotometer $(1.0 \mathrm{~cm}$ quartz cells) was used for all absorbance measurements.

Oxygen saturation and oxygen content were measured using Radiometer OSM3; Hemoximeter adjusted for rat blood.

\section{Oxidative stress parameters:}

\section{A. Determination of superoxide dismutase (SOD) activity}

The activity of SOD was also measured spectrophotometrically at the same wavelength $(540 \mathrm{~nm})$ as described by Boriskin et al. [30]. Briefly, the blood serum was centrifuged in for $10 \mathrm{~min}$ at $6000 \mathrm{rpm}$. Afterwards, we added phosphate buffer $(\mathrm{pH} 7.4)$ to the supernatant with ration 3:1. Then, we centrifuged at $5000 \mathrm{rpm}$ for 15 minutes, precipitated the hemoglobin using 2:1 chloroform: methanol solution with a ratio 2:1 for 10 minutes, centrifuged the reaction mixture, diluted with phosphate buffer 20 times and incubated the miture for 10 minutes with $57 \mu \mathrm{m}$ nitro blue tetrazolium (NBT), $98.5 \mu \mathrm{m}$ NAD $\cdot \mathrm{N}$ and $16 \mu \mathrm{m}$ fenasintrasalud (PMS) in a $0.5 \mathrm{M}$ phosphate buffer with EDTA ( $\mathrm{pH} 8.3$ ) at a temperature of $25^{\circ} \mathrm{C}$ in aerobic conditions. The activity of SOD was calculated by the formula [30]:

Enzymatic Activity $=\frac{\text { Pecentageofinhibitionof } H B \text { Treaction } \%}{100 \%-\text { Percentageofinhibitiono } H \text { HTreductionreaction } \%}$

\section{B. Determination of catalase (CAT) activity}

It was done using the standard method of Boriskin et al. [31]. Briefly, we stated ca reaction of $0.1 \mathrm{ml}$ of blood serum to $2 \mathrm{ml}$ of $0.03 \%$ hydrogen peroxide solution and leave for 10 minutes. Afterwards, we stopped the reaction with $1 \mathrm{ml}$ of $4 \%$ ammonium molybdenum. The Color intensity was measured spectrophotometrically at a wavelength of $410 \mathrm{~nm}$.

Catalase activity is calculated by formula [31]: 
Catalase activity $=\frac{\text { Opticaldensityoftheblank }- \text { Opticaldensityofthesample }}{\text { Volumeofthesample } * \text { timeofincubation } * 1.6 * 100000}$

\section{Determination of malondialdehyde (MDA)}

The amount of MDA was measured as previously mentioned in Hamdan et al [32]. Briefly, we added trichloroacetic acid to the serum in order to precipitate all proteins. Then, we added thiobarbituric acid in order to form thiobarbituric acid. Then, we measured the color intensity at wavelength of $532 \mathrm{~nm}$.

\section{Determination of nitrous oxide (NO) level}

The serum NO concentration was measured indirectly spectrophotometrically in two-step process. Initially, we determined the total serum NO for both nitrate and nitrite concentrations (termed NOx) using the previously mentioned method in Csonka et al [33]. Briefly, we added modified Griess's reagent (1\% sulphanilamide and $0.1 \%$ naphthylethylenediamine in $5 \%$ phosphoric acid) to the serum for 10 min till developing of a deep purple azo mixture and the absorbance was measured at $546 \mathrm{~nm}$. Then, we subtracted the directly measured serum nitrite from the measured total nitrite nitrate concentration to calculate the serum nitrate concentration.

\section{E. Determination of GSH-Px activity in plasma}

We measured the serum activity of GSH-Px also spectrophotometrically at $412 \mathrm{~nm}$ as described by Beevi et al. [34]. Briefly, We added $171 \mathrm{mM}$ of $\mathrm{K}_{2} \mathrm{HPO}_{4} / \mathrm{KH}_{2} \mathrm{PO}_{4}, 4.28 \mathrm{mM}$ of NaN3, $2.14 \mathrm{mM}$ of EDTA, $6 \mathrm{mM}$ of reduced $\mathrm{GSH}, 0.9 \mathrm{mM}$ of $\mathrm{NADPH}$, and $2 \mathrm{U} . / \mathrm{mL}$ of glutathione reductase. We added $50 \mu \mathrm{l}$ of reaction mixture contains $5 \mathrm{mg}$ of $5,5^{\prime}$,dithiobisnitrobenzoic acid in $5 \mathrm{~mL}$ assay buffer stored on ice to form a complex.

\section{F. Determination of Serum iron and total iron binding capacity (TIBC)}

Serum iron and TIBC were measured using immunoassay studies which were carried out using semi-auto analyzer Beckman Coulter AU480.

\section{Cell culture}

We homogenized $100 \mathrm{mg}$ frozen myocardium of each rat in each group of rats in $1 \mathrm{ml}$ of TRIzol ${ }^{B}$ (Thermo Fisher Scientific, Inc., USA). We performed all the processes for total RNA extraction according to the manufacturer's protocol. Total RNA concentration was adjusted spectrophotometrically at a wavelength of $260 \mathrm{~nm}$ using nuclease-free water. Real Time was performed using a high capacity cDNA reverse transcription kit (Thermo Fisher Scientific, Inc., USA) as directed in the manufacturer's protocol.

\section{Determination of gene expression of $n r f 2, \mathrm{NF}-\mathrm{kB}$ and caspase-3}


All genetic expression of the following genes; $n r f 2, N F-K B$ and caspase-3 were assayed as described previously by our group [35]. We used the following sequences of the primers: for nrf2, we used the forward primer: 5'-GAGACGGCCATGACTGAT-3' and the reverse primer: 5'-GTGAGGGGATCGATGAGTAA-3', for NF-KB, we used the forward primer: 5'-ACCCTGACCTTGCCTATTTG-3' and the reverse primer: 5'GAAAAGCTGTAAACATGAGCCG-3', for caspase-3, we sued the forward primer: 5'CGTCTGTGCTCCAGGCTTC-3' and the reverse primer: 5'-TGTGAGTTCCTTCCTTTCTTTGTG-3' and for GAPDH, we used the forward primer: 5'-CCATCAACGACCCCTTCATT-3' and the reverse primer: $5^{\prime}-$ CACGACATACTCAGCACCAGC-3'.

\section{Statistical analysis}

All results were shown as mean \pm standard error of mean (SEM). We used one-way analysis of variance (ANOVA). It was preceded by Tukey's post hoc test using GraphPad Prism ${ }^{\circledR}$ software version 5 . For all analysis, the level of statistical significance was set at $P<0.05$.

\section{Results}

\section{Effect of continuous administration of GTNs on the hematological parameters}

A significant increase in methemoglobin and carboxyhemoglobin, after continous exposure to ISMN to rats $(p<0.05)$ in a concentration-dependent manner comparing to the control group (Fig. 1A, 1B). Meanwhile, continuous administration of ISMN to rats leads to decrease of oxygen saturation, and oxygen content, hemoglobin content $(p<0.05)$ in a concentration-dependent manner (Fig. 1C, 1D). Concomitant administration of the L-ascorbic acid leads to decrease of the increased levels of methemoglobin and carboxyhemoglobin and to restoring of the decreased level of the increased levels of the hemoglobin content, oxygen saturation, and oxygen content for the ISMN treated groups.

\section{Effect of continuous exposure to organic nitrates on the oxidative stress parameters}

Continuous exposure of rats to ISMN leads to a significant increase in the level of the Malondialdehyde (MDA) activity $(p<0.05)$ in a concentration-dependent manner (Fig. 2A). Meanwhile, continuous exposure to ISMN leads to the reduction of the activity of all of the following; Superoxide dismutase (SOD), catalase activity (CAT), Glutathione (GSH) and Nitrous Oxide (NO) content level $(p<0.05)$ in a concentration-dependent manner (Fig. 2B). Concomitant administration of L-ascorbic acid leads to restoring the increased level of MDA and to ameliorating the decreased levels of Superoxide dismutase (SOD), catalase activity (CAT), Glutathione (GSH) and Nitrous Oxide (NO) content for the ISMN treated group (Figure 2). 


\section{Effect of continuous administration of organic nitrates on the iron content, total iron binding capacity and inorganic phosphorus content}

Continuous administration of organic nitrates to rats leads to reduction of both the serum iron level and total iron binding capacity $(\mathrm{TIBC})(\mathrm{p}<0.05)$ in a concentration-dependent manner. Concomitant administration of L-ascorbic acid leads to restoring the decreased level of the serum iron and the TIBC in rats (Figure 3A, 3B).

\section{Effect of continuous exposure of organic nitrates on the mitochondrial oxidative stress marker ( $n r f 2$ ), the pro- inflammatory cytokines marker ( NF-KB) and apoptosis markers ( caspase-3) concentrations in myocardium muscles}

Chronic exposure to organic nitrates caused a significant reduction of the genetic expression of nrf2 in myocardium muscle tissues in a concentration-dependent manner. However, vitamin $\mathrm{C}$ restored the normal level of $n r f 2$ in the myocardium tissue (Fig. 4A). Moreover, Continuous exposure of rats to organic nitrates led to a significant increase of the genetic expression of nuclear factor NF-KB and caspase- 3 in myocardium muscle tissues in a concentration-dependent manner (Fig. 4B, 4C). However, vitamin C restored the level of NF-KB and caspase-3 in the myocardium tissue.

\section{Discussion}

The act of nitric oxide (NO) is multifaced. It can be as a messenger for either a pro-oxidant or an antioxidant in biological systems [35]. All organic nitrates undergo biotransformation releasing nitric oxide (NO) [34]. Chronic exposure of nitrates induces oxidative stress and exerts disastrous pathophysiological effects including oxidative stress, inflammation, autoimmune disease and cancer [35]. This may be directly due to potential liability of mediating DNA damage or indirectly through production of reactive nitrogen species (RNS) free radicals. NO has short half-life and widely formed through the vascular endothelium, resulting in direct relief of the smooth muscles of the vascular tissues [36]. Serum level of both Nitates and Nitrites were used to estimate the level of NO due to its very short half-life [33]. We chose the rat model since rats are more similar to human physiology over mice model, making them better models for the studying pathological conditions in pre-clinical trials [37, 38]. Moreover, high sucrose/fat diet (HSF) induces hypertension rats; called spontaneously hypertensive rats (SHR) which are extensively used as an in vivo model for essential hypertension and cardiovascular 
disease [27]. This SHR rat model has elevated basal myocardial NO content which may be due to an increase in the protein-bound of dinitrosyl nonheme iron complexes which liberates the NO to the peripheral circulation that helps in treating the hypertensive state. Moreover, it has been previously published that SHR rats showed increased NO synthase activity (NOS) III in the cardiac and aortic endothelia. Both of these two enzymes helps in regulating the vasoreactivity in the SHR rats [27].

Our data go along with the previously published data for using antioxidant for treating the nitrate tolerance and they gave similar clinical data (34,38-39). Meanwhile, the authors stressed on the oxidative stress markers. Here, we studied a very important stress marker; nrf2, has a great role in the antioxidant metabolism pathway and has important role against reactive oxygen species producing the cellular injury in the myocardium muscles [40]. This gives a molecular explanation of the previously published results and quantitatively determined the dose of vitamin $C$ that can be used clinically. Moreover, we studied the activity of pro-inflammatory mediator gene; NF-kB. Our results showed another mechanism for the protective effect of the vitamin c against NO deleterious effect on the myocardium tissue. Besides, our data on the apoptosis marker; caspase-3, gives the an explanation for the end result of the protective effect of vitamin $\mathrm{c}$ on the myocardium tissue.

SOD, GSH, CAT and MDA are the first line defense mechanisms against oxidative stress. They give an indication about the stressful conditions inside the cells [36]. It has been previously reported a significant decrease in the activity of SOD, GSH and CAT, and a significant elevated level of plasma MDA [27]. The suggested mechanism is that this may be an indirect effect of the significant increased NO plasma level or directly due to the significant elevated level of ROS and RNS. Meanwhile, the previous study [27] has not proved the tightly bound to that hypothesized subcellular event. In our study, we proved these suggestions through finding the dose-dependent response according to the plasma NO levels. Yet, vitamin C; a free radical scavenger, restored the change of these first line defense mechanisms against oxidative stress. We measured the cellular levels of pro-inflammatory cytokines marker (NF-KB), apoptosis markers (caspase-3) and mitochondrial oxidative stress marker ( $n r f 2$ ) in myocardium muscles. $n r f 2$ is a suppressive mitochondrial oxidative stress marker. If this marker is increased, this indicates an increased oxidative stress conditions inside the cell. We found that ISMN elevated the cellular activity of nrf 2 in a concentration-dependent manner. Moreover, ISMN reduced the cellular activity of both proinflammatory cytokines marker (NF-KB) and apoptosis markers (caspase-3) in a dose-dependent manner. Vitamin $\mathrm{C}$ succeeded to restore the cellular levels of the three markers in all tested concentrations of ISMN. We expected that the vasodilatory effect of ISMN will remain in chronic administration of ISMN due to the restoring of the endothelial function of myocardium and no need to establish the NO free period. This nitrate free period for patients that may cause elevation of the risk of the cardiovascular events during such nitrate-free periods, specifically in the early light phase.

Reduction of the plasma hemoglobin level and increase in the level of both methemoglobin and carboxyhemoglobin may be the consequences of the liberated nitric oxide and nitrate ions during isosorbide mononitrate metabolism which can oxidize hemoglobin to methemoglobin and carboxyhemoglobin $[39,40]$. Increased methemoglobin level can cause congenital enzymatic defects, 
variation in hemoglobin molecule [41, 42]. Methemoglobin does not bind to oxygen efficiently causing a reduction in the oxygen carrying capacity of the blood and reduction in saturated oxygen and oxygen content this decrease is significant in high doses of nitrates [43]. Vitamin C protects the blood from the oxidant effect of nitric oxide (antioxidant effect) and induces decreasing of level of met-hemoglobin concentration [42]. This reduction in methemoglobin levels induced by vitamin $\mathrm{C}$ concluded that erythrocyte alone had a negligible ability to reduce methemoglobin in the absence of exogenous ascorbate. Ascorbic acid preserves the $\mathrm{Hb}$ in a reduced ferrous redox state $[43,44]$.

It has been previously reported that carboxyhemoglobin showed a significant increase with high doses of organic nitrates only [45]. Our results showed that low doses of ISMN can significantly increase the plasma level of MET-Hb. This may be due to the impairment in the antioxidant enzymes defense system of the erythrocyte which may cause an elevation of the abnormal hemoglobin derivatives as carboxyhemoglobin [46]. An elevation in carboxyhemoglobin levels were noticed in all ISNN treated groups in a concentration-dependent manner. However, following vitamin $\mathrm{C}$ administration, a significant reduction of the carboxyhemoglobin was obtained in all treated groups. A reduction in hematocrit, RBC, and WBC may be a result of hemoglobin reduction and oxidative stress induced by nitric oxide $[46,47]$.

The present study showed a significant decrease in both serum iron level and serum total iron binding capacity (TIBC) in all ISMN treated groups in a concentration-dependent manner. This result can be explained by the increased serum nitric oxide by the increased ISMN dose and by the induced oxidative stress decreasing the serum iron and total iron binding capacity (TIBC) [48]. It has been reported that iron deficiency anemia increases through NO production, and elevated NO concentrations in iron deficiency anemia. This effect can be revered by iron supplementation to regain its normal levels [47]. In this study an improvement of the serum iron level and total iron binding capacity (TIBC) was observed by the effect of vitamin C. This improvement may be due to the beneficial effect of vitamin $C$ to enhance iron absorption $[47,48]$. In our study all groups treated with ISMN showed a significant increase in serum nitric oxide concentration. It has been previously recorded that vitamin $\mathrm{C}$ significantly decrease nitric oxide concentration .

Vitamin $\mathrm{C}$ can decrease the accumulation of superoxide and peroxynitrite by directly scavenging superoxide [45]. Vitamin $C$ safeguards against oxidative stress that induces pathological vasoconstriction and destruction of the endothelial barrier through blocking tetrahydrobiopterin $\left(\mathrm{BH}_{4}\right)$ oxidation, the cofactor of the endothelial nitric oxide synthase (eNOS), thereby inhibiting endothelial nitric oxide (eNO) depletion and eNOS uncoupling. Ascorbate blocks inducible nitric oxide synthase (iNOS) preventing abundant production of NO [49].

\section{Conclusion}

Our results explores the beneficial effects of ascorbic acid in nitrate therapy. Nitrates induced oxidative stress exerts disastrous effects. Ascorbic acid supplementation could emerge as an important therapeutic strategy to prevent organic nitrate oxidative stress and can reduce nitrate tolerance. 


\section{Declarations}

\section{Ethical approval}

All animal procedures were reviewed and got the approval by the ethical research projects committee in University of Tabuk (UT-46-10-2018) and all steps were performed in accordance with its relevant standard guidelines and regulations.

\section{Consent to Participate}

All authors read and approve the manuscript and agree to participate in the research.

\section{Consent to publish}

All authors have read and approved the final manuscript.

\section{Competing interests}

All authors declare no potential conflicts of interest, including any financial, personal or other relationships with other people or organizations within that could inappropriately influence, or be perceived to influence, this work.

\section{Author contribution}

AMEH; paper drafting the article and revising it critically for important intellectual content, ZMM; supervised and monitored all aspects of this study from conception of the idea to submission of the manuscript, AA; contributed her statistical analysis and data interpretation, SMHE; contributed by the intellectual ability to conception the project design. All authors contributed equally to the final version of the manuscript. All authors read and approve the manuscript and all data were generated in-house and that no paper mill was used.

\section{Funding}

This research is neither fully nor partially funded by an organization.

The authors declare that this manuscript is not published or communicated for publication elsewhere either in part or full.

\section{Competing interest}

The authors declare that they have no competing interests.

\section{Availability of data and materials}

All data are available on request. 


\section{References}

1. Gori T, Parker JD (2004) Long-term therapy with organic nitrates: the pros and cons of nitric oxide replacement therapy. J Am Coll Cardiol 44:632-634. https://doi:10.1016/j.jacc.2004.05.031

2. Steven S, Oelze M, Hausding M et al (2018) The Endothelin Receptor Antagonist Macitentan Improves Isosorbide-5-Mononitrate (ISMN) and Isosorbide Dinitrate (ISDN) Induced Endothelial Dysfunction, Oxidative Stress, and Vascular Inflammation. Oxid Med Cell Longev 2018:7845629. https://doi:10.1155/2018/7845629

3. Daiber A, Oelze M, Coldewey M et al (2004) Oxidative stress and mitochondrial aldehyde dehydrogenase activity: a comparison of pentaerythritol tetranitrate with other organic nitrates. Mol Pharmacol 66:1372-1382. https://doi:10.1124/mol.104.002600

4. Sydow K, Hornig B, Arakawa N, Bode-Böger SM, Tsikas D, Münzel T, Böger RH (2004) Endothelial dysfunction in patients with peripheral arterial disease and chronic hyperhomocysteinemia: potential role of ADMA. Vasc Med 9:93-101. https://doi:10.1191/1358863x04vm538oa

5. Mullane D, Lenihan M, Hanley C, Wall T, Bukowska I, Griffin M, Flood G (2020) Efficacy of Glyceryl trinitrate (GTN) to facilitate the rewarming process during cardiopulmonary bypass. J Cardiothorac Surg 15(1):214. https://doi.org/10.1186/s13019-020-01258-0

6. Abrams J (1996) Beneficial actions of nitrates in cardiovascular disease. Am J Cardiol 77:31C-7C. https://doi:10.1016/s0002-9149(96)00186-5

7. Knuuti J, Wijns W, Saraste A et al (2019) Scientific Document Group 2019 ESC Guidelines for the diagnosis and management of chronic coronary syndromes: The Task Force for the diagnosis and management of chronic coronary syndromes of the European Society of Cardiology (ESC). Eur Heart J 41(3):407-477. https://doi:10.1093/eurheartj/ehz425

8. Daiber A, Münzel T (2015) Organic Nitrate Therapy, Nitrate Tolerance, and Nitrate-Induced Endothelial Dysfunction: Emphasis on Redox Biology and Oxidative Stress. Antioxid Redox Signal 23:899-942 https://doi:10.1089/ars.2015.6376

9. Ivy JL (2019) Inorganic Nitrate Supplementation for Cardiovascular Health. Methodist Debakey Cardiovasc J 15:200-206. https://doi:10.14797/mdcj-15-3-200

10. Thatcher GR, Nicolescu AC, Bennett BM, Toader V (2004) Nitrates and NO release: contemporary aspects in biological and medicinal chemistry. Free Radic Biol Med. 37:1122-1143. https://doi:10.1016/j.freeradbiomed.2004.06.013

11. Awad MA, Aldosari SR, Abid MR (2018) Genetic Alterations in Oxidant and Anti-Oxidant Enzymes in the Vascular System. Front Cardiovasc Med 5:107. https://doi:10.3389/fcvm.2018.00107

12. Marković SD, Dorđević NZ, Curčić MG, Stajn AS, Spasić MB (2014) Biotransformation and nitroglycerin-induced effects on antioxidative defense system in rat erythrocytes and reticulocytes. Gen Physiol Biophys 33:393-401. https://doi:10.4149/gpb_2014018

13. Sydow K, Daiber A, Oelze M, et al (2004) Central role of mitochondrial aldehyde dehydrogenase and reactive oxygen species in nitroglycerin tolerance and cross-tolerance. J Clin Invest 113:482-489. 
https://doi:10.1172/JCl19267

14. Axton ER, Cristobal E, Choi J, Miranda CL, Stevens JF (2018) Metabolomics-Driven Elucidation of Cellular Nitrate Tolerance Reveals Ascorbic Acid Prevents Nitroglycerin-Induced Inactivation of Xanthine Oxidase. Front Pharmacol 9:1085. https://doi:10.3389/fphar.2018.01085

15. Azevedo ER, Schofield AM, Kelly S, Parker JD (2001) Nitroglycerin withdrawal increases endotheliumdependent vasomotor response to acetylcholine. J Am Coll Cardiol 37:505-509. https://doi:10.1016/s0735-1097(00)01140-2

16. Cosby K, Partovi KS, Crawford JH et al Nitrite reduction to nitric oxide by deoxyhemoglobin vasodilates the human circulation. Nat Med 9:1498-1505. https://doi:10.1038/nm954

17. Tai YH, Chu YH, Wu HL, Lin SM, Tsou MY, Huang CH, Chang H, Lu C (2020) High-dose Nitroglycerin Administered during Rewarming Preserves Erythrocyte Deformability in Cardiac Surgery with Cardiopulmonary Bypass. Microcirculation 27(4):e12608. https://doi:10.1111/micc.12608

18. Hathazi D, Scurtu F, Bischin C, Mot A, Attia AAA, Kongsted J, Silaghi-Dumitrescu R (2018) The Reaction of Oxy Hemoglobin with Nitrite: Mechanism, Antioxidant-Modulated Effect, and Implications for Blood Substitute Evaluation. Molecules 23(2):350. https://doi:10.3390/molecules23020350

19. Wang D, Piknova B, Solomon SB et al. In vivo reduction of cell-free methemoglobin to oxyhemoglobin results in vasoconstriction in canines. Transfusion 53:3149-163. https://doi:10.1111/trf.12162

20. Ash-Bernal R, Wise R, Wright SM (2004) Acquired methemoglobinemia: a retrospective series of 138 cases at 2 teaching hospitals. Medicine (Baltimore) 83:265-273. https://doi:10.1097/01.md.0000141096.00377.3f

21. Rother RP, Bell L, Hillmen P, Gladwin MT (2005) The clinical sequelae of intravascular hemolysis and extracellular plasma hemoglobin: a novel mechanism of human disease. JAMA 293:1653-1662. https://doi:10.1001/jama.293.13.1653

22. Minneci PC, Deans KJ, Zhi H, Yuen PS, Star RA, Banks SM et al (2005) Hemolysis-associated endothelial dysfunction mediated by accelerated $\mathrm{NO}$ inactivation by decompartmentalized oxyhemoglobin. J Clin Invest 115:3409-3417. https://doi:10.1172/JCl25040

23. Schaer DJ, Alayash Al (2010) Clearance and control mechanisms of hemoglobin from cradle to grave. Antioxid Redox Signal 12:181-184. https://doi:10.1089/ars.2009.2923

24. Jurt U, Gori T, Ravandi A, Babaei S, Zeman P, Parker JD (2001) Differential effects of pentaerythritol tetranitrate and nitroglycerin on the development of tolerance and evidence of lipid peroxidation: a human in vivo study. J Am Coll Cardiol 38:854-859. https://doi:10.1016/s0735-1097(01)01414-0

25. Doseděl M, Jirkovský E, Macáková K et al (2021) Vitamin C-Sources, Physiological Role, Kinetics, Deficiency, Use, Toxicity, and Determination. Nutrients 13(2):615. https://doi:10.3390/nu13020615

26. Cartaya A, Maiocchi S, Bahnson EM (2019) Nanotherapies for Treatment of Cardiovascular Disease: A Case for Antioxidant Targeted Delivery. Curr Pathobiol Rep 7(3):47-60. https://doi:10.1007/s40139019-00196-4 
27. Plotnick MD, D'Urzo KA, Gurd BJ, Pyke KE (2017) The influence of vitamin C on the interaction between acute mental stress and endothelial function. Eur J Appl Physiol 117(8):1657-1668. https://doi:10.1007/s00421-017-3655-4

28. Li T, Bai B, Tian C, Wang H, Jiang D, Ma F, Shan M (2018) High sucrose/fat diet and isosorbide mononitrate increase insulin resistance, nitric oxide production and myocardial apoptosis in a hypertensive rat model. Molecular Medicine Reports 17(5):6789-6795. https://doi:10.3892/mmr.2018.8651

29. Arnaud F, Higgins A, McCarron R, Moon-Massat PF (2017) Determination of methemoglobin and hemoglobin levels in small volume samples. Artif Cells Nanomed Biotechnol 45(1):58-62. https://doi:10.3109/21691401.2016.1138490

30. Boumba VA, Vougiouklakis T (2005) Evaluation of the Methods Used for Carboxyhemoglobin Analysis in Postmortem Blood. Int J Toxicol 24(4):275-281. https://doi:10.1080/10915810591007256

31. Carolina Rosa A, Corsi D, Niccolò Cavi, Bruni N, Dosio F (2021) Superoxide Dismutase Administration: A Review of Proposed Human Uses. Molecules 26(7):1844. https://doi:10.3390/molecules26071844

32. Boriskin P, Gulenko O, Deviatkin A, Pavlova O, Toropovskiy A (2019) Correlation of superoxide dismutase activity distribution in serum and tissues of small experimental animals. IOP Conference Series. Earth and Environmental Science 403: 012113. https://doi:10.1088/17551315/403/1/012112

33. Csonka C, Páli T, Bencsik P, Görbe A, Ferdinandy P, Csont T (2015) Measurement of NO in biological samples. Br J Pharmacol 172(6): 1620-1632. https://doi:10.1111/bph.12832

34. Beevi SSS, Rasheed AMH, Geetha A (2004) Evaluation of oxidative stress and nitric oxide levels in patients with oral cavity cancer. Jpn J Clin Oncol 34(7):379-385. https://doi:10.1093/jjco/hyh058

35. Hamdan AM, Al-Gayyar MM, Shams MEE, Alshaman US, Prabahar K, Bagalagel A, Diri R, Noor AO, Almasri D (2019) Thymoquinone therapy remediates elevated brain tissue inflammatory mediators induced by chronic administration of food preservatives. Sci Rep 9:7026. https://doi.org/10.1038/s41598-019-43568-x

36. Kumar V, Gill KD (2018) Estimation of Inorganic Phosphorus in Serum and Urine. In: Basic Concepts in Clinical Biochemistry: A Practical Guide. Springer, Singapore. https://doi.org/10.1007/978-981-108186-6_22

37. Chen Z, Zhang J, Stamler JS (2002) Identification of the enzymatic mechanism of nitroglycerin bioactivation. Proc Natl Acad Sci U S A 99:8306-8311. https://doi:10.1073/pnas.122225199

38. Lu L, Rao X, Cong R, Zhang C, Wang Z, Xu J, Tanabe G, Muraoka O, Wu X, Xie W. (2019) Design, Synthesis and Biological Evaluation of Nitrate Derivatives of Sauropunol A and B as Potent Vasodilatory Agents. Molecules 24(3):583. https://doi:10.3390/molecules24030583

39. Costa D, Benincasa G, Lucchese R, Infante T, Nicoletti GF, Napoli C (2019) Effect of nitric oxide reduction on arterial thrombosis. Scand Cardiovasc J 53:1-8. https:// 
https://doi.org/10.1080/14017431.2019.1581943

40. Bryda EC (2013) The Mighty Mouse: the impact of rodents on advances in biomedical research. Mo Med 110:207-211.

41. Iannaccone PM, Jacob HJ (2009) Rats!. Dis Model Mech 2:206-210. https://doi:10.1242/dmm.002733

42. Amdahl MB, DeMartino AW, Gladwin MT (2019) Inorganic nitrite bioactivation and role in physiological signaling and therapeutics. Biol Chem 401:201-211. https://doi:10.1515/hsz-20190349

43. Rochon ER, Missinato MA, Xue J, Tejero J, Tsang M, Gladwin MT, Corti P (2020) Nitrite Improves Heart Regeneration in Zebrafish. Antioxid Redox Signal 32(6):363-377. https://doi:10.1089/ars.2018.7687

44. Ashurst J, Wasson M. Methemoglobinemia: a systematic review of the pathophysiology, detection, and treatment. Del Med J. 2011;83:203-208.

45. Schechter AN, Gladwin MT (2003) Hemoglobin and the paracrine and endocrine functions of nitric oxide. N Engl J Med 348:1483-1485. https://doi:10.1056/NEJMcibr023045

46. Simoni J, Villanueva-Meyer J, Simoni G, Moeller JF, Wesson DE (2009) Control of oxidative reactions of hemoglobin in the design of blood substitutes: role of the ascorbate-glutathione antioxidant system. Artif Organs 33:115-126. https://doi:10.1111/j.1525-1594.2008.00695.x

47. Waugh WH (2008) Inhibition of iron-catalyzed oxidations by attainable uric acid and ascorbic acid levels: therapeutic implications for Alzheimer's disease and late cognitive impairment. Gerontology 54:238-243. https://doi:10.1159/000122618

48. Oudemans-van SHM, Spoelstra-de MAM, de WMC (2014) Vitamin C revisited. Crit Care 18:460. https://doi:10.1186/s13054-014-0460-x

49. May JM, Qu ZC (2011) Nitric oxide mediates tightening of the endothelial barrier by ascorbic acid. Biochem Biophys Res Commun 404:701-705. https://doi:10.1016/j.bbrc.2010.12.046

\section{Figures}


Figure 1

A

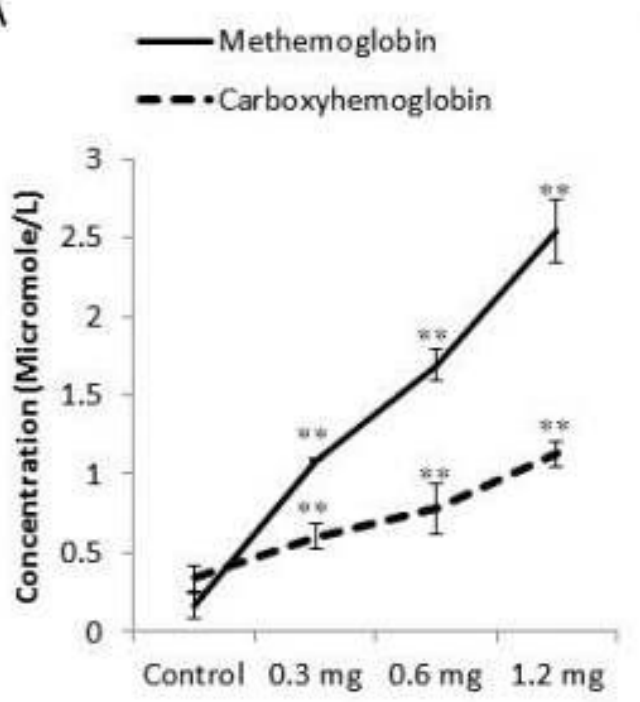

C

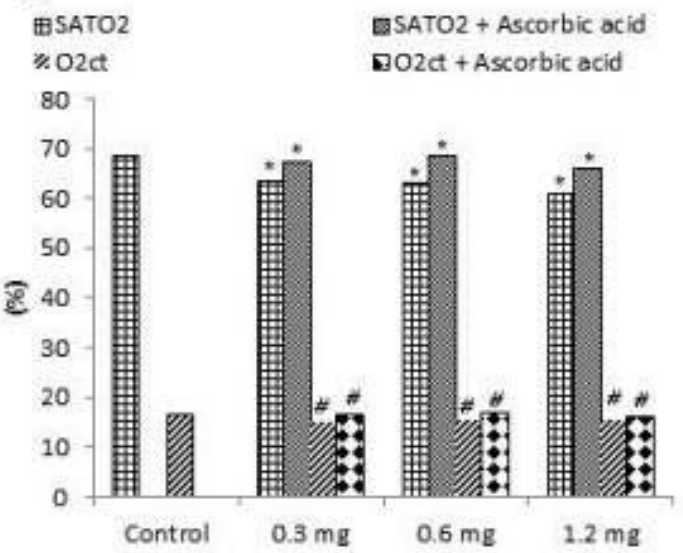

B mMethemoglobin

용 Methemoglobin + Ascorbic acid

口Carboxyhe mog lobin

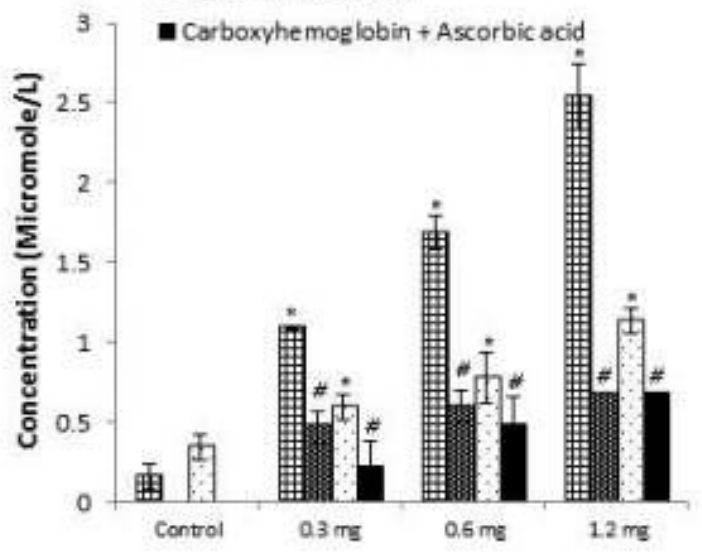

D

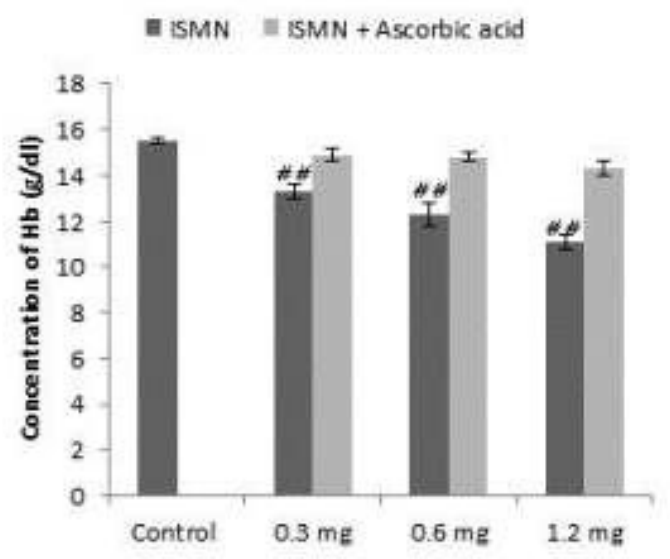

\section{Figure 1}

Effect of continuous administration of GTNs on the hematological parameters Data represented as means $\pm S D$. $n=5-6 *$ : Significant decrease compared with control groups at $p<0.05$. $* *$ : Significant increase compared with control groups at $p<0.05$. \#: Significant decrease compared with ISMN groups of the same dose at $p<0.05$. \#\#: Significant increase compared with ISMN groups of the same dose at $p<0.05$. (A) Methemoglobin and Carboxyhemoglobin serum levels are increased by continuous 
administration of ISMN in a concentration-dependent manner. (B) Continuous administration of ISMN increased the serum levels of Methemoglobin and Carboxyhemoglobin $(p<0.05)$ in a concentrationdependent manner. Concomitant administration of L-ascorbic acid decreases their increased serum levels. (C) Continuous administration of ISMN decreased of oxygen saturation and oxygen content $(\mathrm{p}<$ 0.05) in a concentration-dependent manner. Concomitant administration of L-ascorbic acid restored the oxygen saturation and oxygen content concentrations. (D) Continuous administration of ISMN decreased the hemoglobin content $(p<0.05)$ in a concentration-dependent manner. Concomitant administration of the L-ascorbic acid restored the decreased level of the hemoglobin content. 
Figure 2

A

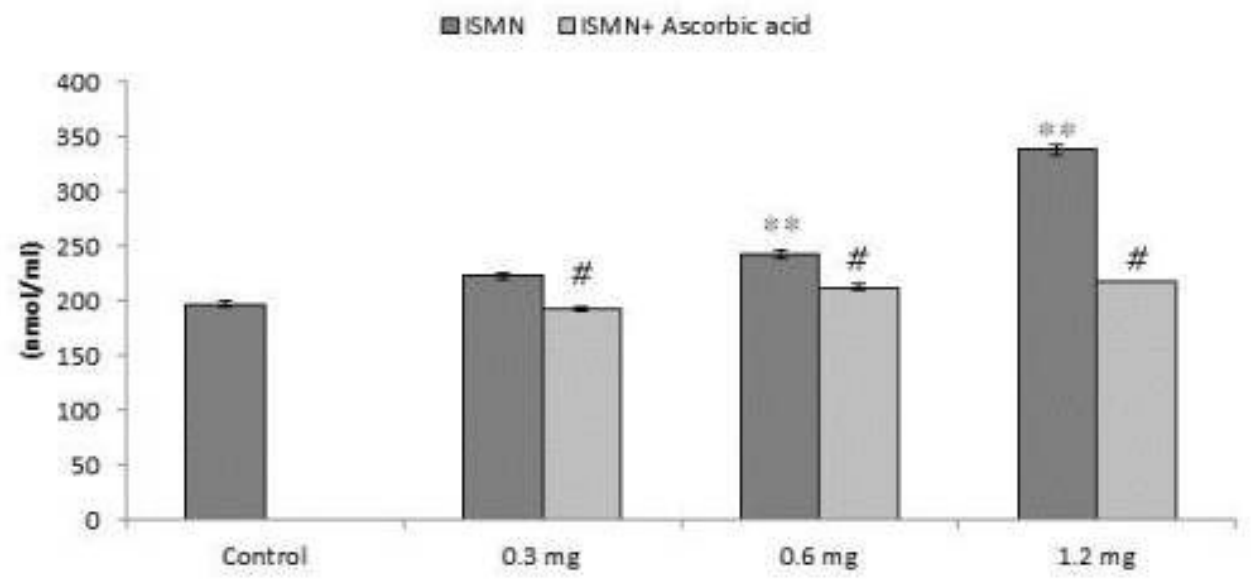

B

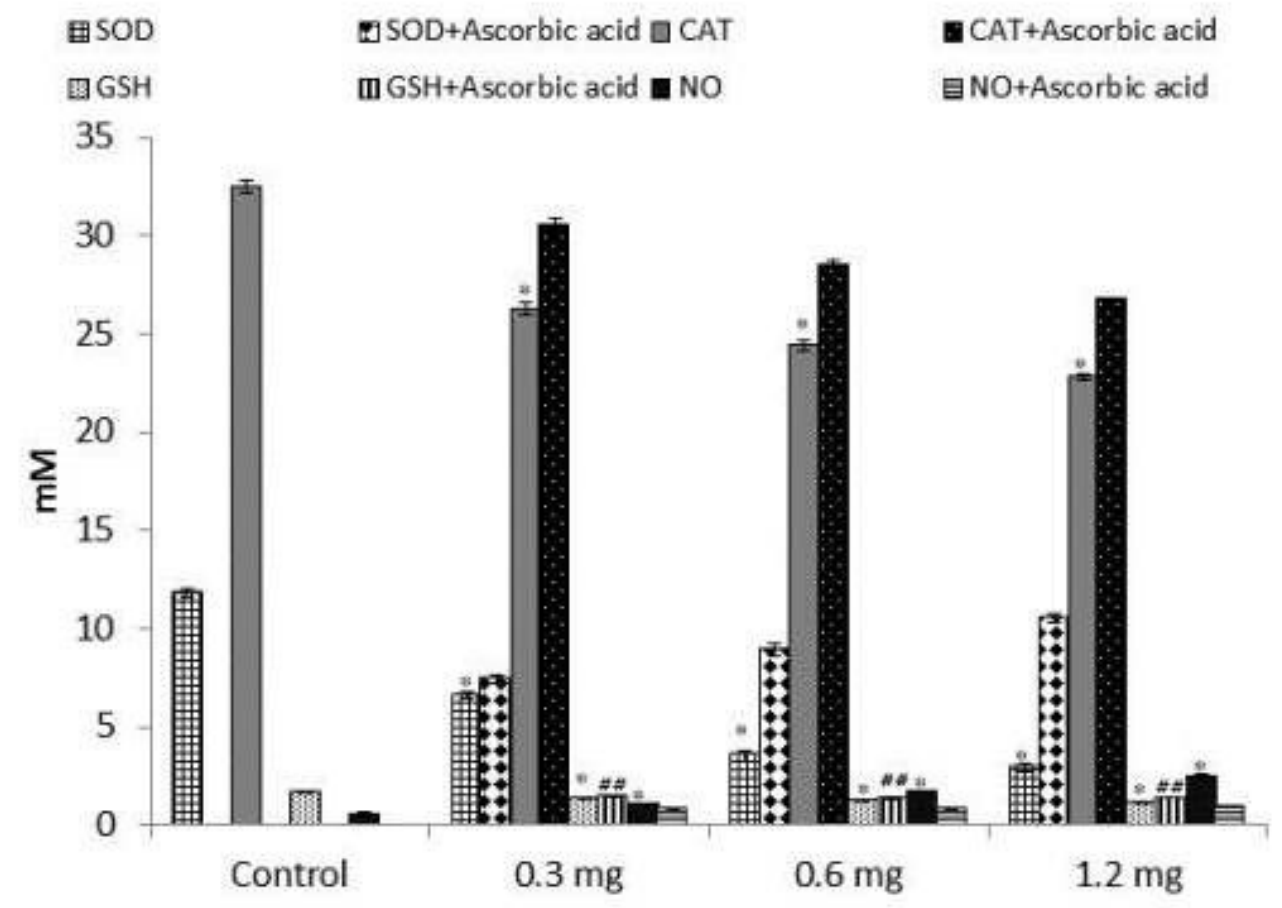

Figure 2

Effect of continuous exposure to organic nitrates on the oxidative stress parameters Data represented as means \pm SD. $n=5-6 *$ : Significant decrease compared with control groups at $p<0.05$. **: Significant increase compared with control groups at $p<0.05$. \#: Significant decrease compared with ISMN groups of the same dose at $p<0.05$. \#\#: Significant increase compared with ISMN groups of the same dose at p<0.05. MDA; Malondialdehyde, SOD; Superoxide dismutase, CAT; Catalase activity, GSH; Glutathione, NO; 
Nitrous Oxide (A) Continuous exposure to ISMN increased Malondialdehyde (MDA) activity $(p<0.05)$ in a concentration-dependent manner. Concomitant administration of L-ascorbic acid leads to restoring the increased level of MDA (B) Continuous exposure to ISMN leads decreased the activity of Superoxide dismutase (SOD), catalase activity (CAT), Glutathione (GSH) and Nitrous Oxide (NO) content level $(p<$ 0.05 ) in a concentration-dependent manner. Concomitant administrated the decreased levels of Superoxide dismutase (SOD), catalase activity (CAT), Glutathione (GSH) and Nitrous Oxide (NO) content.

Figure 3

A

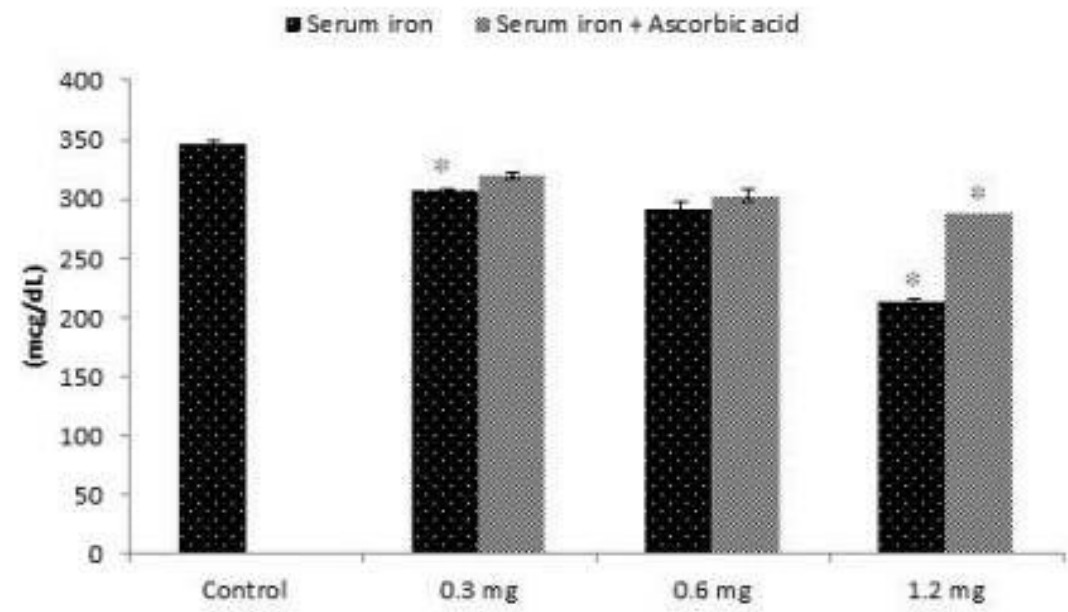

B

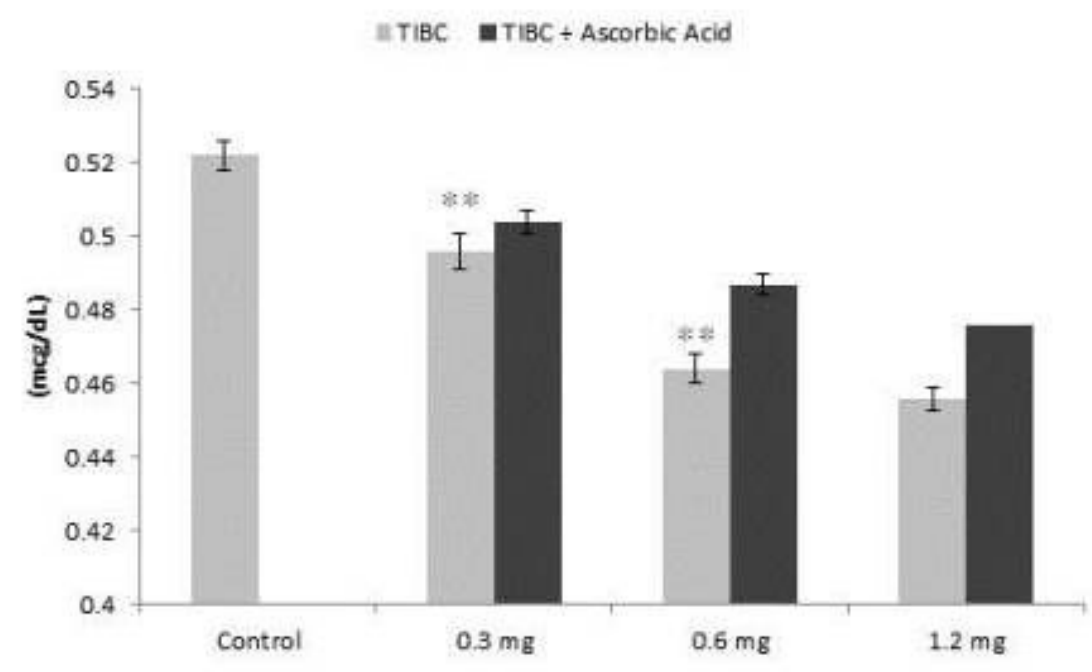

Figure 3 
Effect of continuous administration of organic nitrates on the iron content, total iron binding capacity and inorganic phosphorus content Data was presented as means \pm SD. $n=4-6 *$ : Significant as compared with control group at $p<0.05$. **: Significant as compared with control group at $p<0.05$. (A) Continuous administration of organic nitrates reduced the serum iron level $(p<0.05)$ in a concentration-dependent manner. Concomitant administration of L-ascorbic acid restored the decreased level of the serum iron. (B) Continuous administration of organic nitrates leads to reduction of total iron binding capacity (TIBC) ( $p<$ 0.05) in a concentration-dependent manner. Concomitant administration of L-ascorbic acid leads to restoring the decreased level of the TIBC. 
A

wnf2 =nrf2+Ascorbic acid

Figure 4

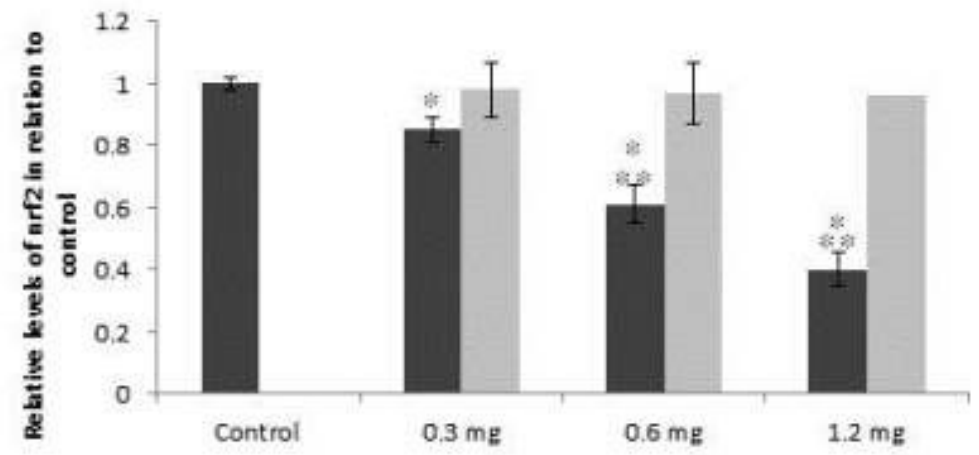

B

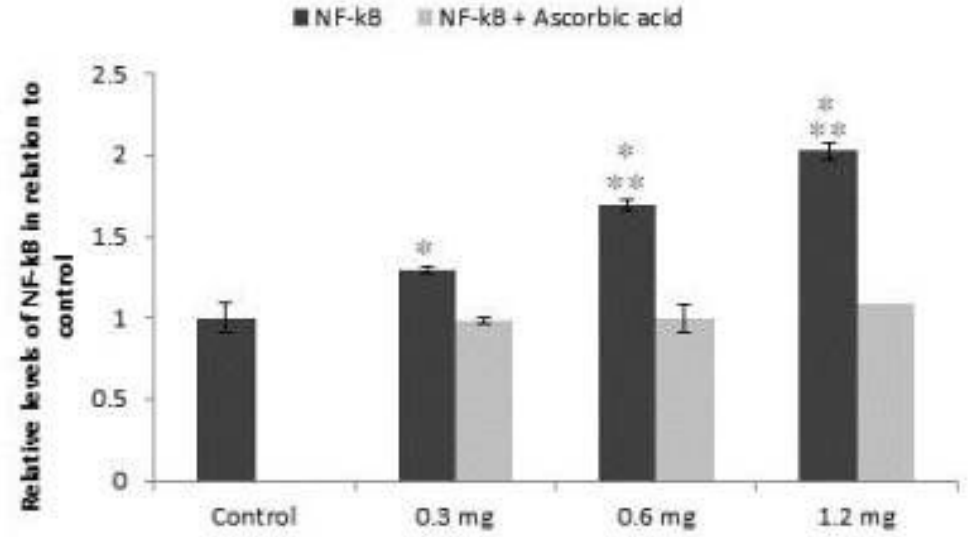

C

- caspase-3 ill caspase-2+ Ascorbic acid

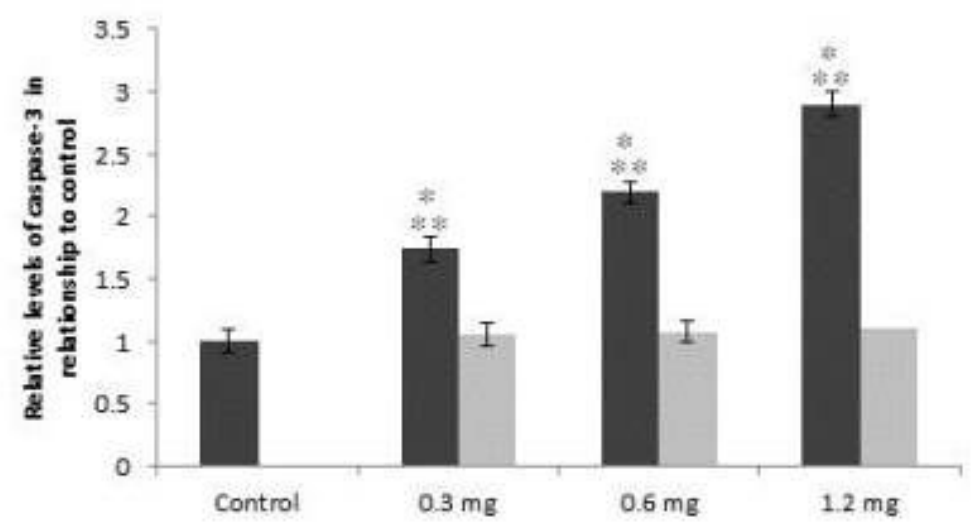

\section{Figure 4}

Effect of continuous exposure of organic nitrates on the mitochondrial oxidative stress marker (nrf2), the pro-inflammatory cytokines marker (NF-kB) and apoptosis markers (caspase-3) concentrations in myocardium muscles Data was presented as means \pm SD. $n=4-6$ *: Significant as compared with control group at $p<0.05$. **: Significant as compared with Ascorbic acid treated group at $p<0.05$. (A) Continuous administration of organic nitrates reduced the relative activity level of nrf2 $(p<0.05)$ in a concentration- 
dependent manner. Concomitant administration of L-ascorbic acid restored the decreased relative activity level of the nrf2. (B) Continuous administration of organic nitrates leads to increase of the relative activity level of NF-kB $(p<0.05)$ in a concentration-dependent manner. Concomitant administration of L-ascorbic acid leads to restoring the increased level of the NF-kB. (C) Continuous administration of organic nitrates leads to increase of the relative activity level of caspase-3 $(p<0.05)$ in a concentration-dependent manner. Concomitant administration of L-ascorbic acid leads to restoring the increased level of the caspase-3.

\section{Supplementary Files}

This is a list of supplementary files associated with this preprint. Click to download.

- Results.xlsx 